\title{
Analysis Of Inertial Stability Dynamical For Robot Based On Lie Algebra
}

\author{
LAI Xin-Xing ${ }^{1, a}$, ZHONG Jin ${ }^{1}$ \\ ${ }^{1}$ Faculty of Science, Jiangxi University of Science and Technology, Ganzhou Jiangxi 341000, China \\ alaixinxing1213@163.com
}

Keywords: Lie Algebra; Robot; Stability Dynamical

\begin{abstract}
In order to improve the speed and accuracy of targets while avoiding the opponent's riposte, this paper presents a method approach to robot trajectory planning which based on Lie algebra and on the background of a high degree of competitive against offensive and defensive network robot system (TLS). There are some key limitations in the Lie algebra planning process: 1.Vision limitations, to maintain the target in one's own camera's field of vision;2.Limitations of avoiding the robot hitting to vital parts from enemy while attacking;3.Kinematic constraints, to make robot avoid reaching the kinematic areas. Meanwhile, it uses the image-based visual servo tracking targets to meet the needs of attack and defense.
\end{abstract}

\section{Introduction}

In mathematics, an affine Lie algebra is an infinite-dimensional Lie algebra that is constructed in a canonical fashion out of a finite-dimensional simple Lie algebra. It is a Kac-Moody algebra for which the generalized Cartan matrix is positive semi-definite and has corank1. From purely mathematical point of view, affine Lie algebras are interesting because their representation theory, like representation theory of finite dimensional, semisimple Lie algebras is much better understood than that of general Kac-Moody algebras. As observed by Victor Kac, the character formula for representations of affine Lie algebras implies certain combinatorial identities, the Macdonald identities[1,2]. Artificial potential field method is a kind of virtual force method proposed by Khatib in 1986, the method regards mobile robot's movement in the environment as a virtual manual movement of the force field, and has a path planning model through environmental potential field. In the artificial potential field method, artificial attractive potential field region represents a target position in Lie algebra, and appropriate exclusion zone represents an obstacle, therefore, the trajectory of reaching the target is associated with one independent flow line which starts from initial point inside gradient field. That means a planning approach which bases on whole-information can produce by tracking this flow line[3,4,5]. Artificial potential field method is relatively simple and the computational complexity is smaller, more suitable for dynamic environments.

\section{Proposed Algorithm}

Robot motion by the impact of artificial potential field, attractive potential field $r_{a}$ approaches the target position of the robot, and Repulsion potential field $r_{n}$ makes the robot away from the edge of the image field, kinematic singularity zones and restricted areas to combat hazardous areas. The force generated by the potential field can be defined as Lie algebra, such as:

$s(q)=-\Delta_{k}^{t} r$

$\mathrm{r} \in \mathrm{R} 6 \times 1$ wherein said robot Cartesian spatial coordinates, represents $r$ gradient value at $q$. In the artificial potential venue, the force on the robot can be expressed as:

$s(q)=\alpha s_{a}(q)+\beta s_{t}(q)=-\alpha \Delta_{k}^{t} r_{t}$

Where $\alpha$ and $\beta$ represent the attraction and Repulsion weights .

This situation can be seen as the result of formula (2) because the equal of attraction and repulsion, at this time, the potential field gradient vector becomes 0 and robots into a stalemate.

For TLS operation, artificial potential field Repulsive force potential field can be defined as: 
$S_{\text {tep }}(q)=\frac{r}{\left\|q_{\text {vital }}(q)-w_{k}\right\|^{2}}, k \in\{1,2,3\}$

Where $\mathrm{r}$ is the current position of the robot pose, $q_{\text {vitali }}(q)$ represents the robot's own $k_{\text {th }}$ laser receiver position(Because of its disk fixed to the end of the robot, and is therefore related to $r$ ), and $w_{k}$ represents the location of the point with shortest distance between enemy laser beam L2 and $q_{\text {vitali }}(q)$, and $\mathrm{k}$ represents Scale Factor.

Consider the following cases, when the robot reach the desired position and orientation (hit enemy key),If the own receiver is close to the enemy laser beam, under the influence of Repulsive force, the location is not the total energy of the global minimum potential field, in TLS offense and defense which causes the own laser beam cannot hit the target.

In order to make the desired position and orientation at the global minimum of the potential field of energy, need to build a new repulsive force function. Reach the desired position and orientation, the robot with the minimum of repulsion. According to the above features to create new function of repulsive force:

$S_{\text {tep }}(q)=\frac{r}{\left\|q_{\text {vitail }}(q)-w_{k}\right\|^{2}}\left\|q-q_{\text {stpp }}\right\|^{2}, k \in\{1,2,3\}$

Among, $\left\|q_{\text {vitail }}\right\|^{2}$ is distance between the robot laser receiver and the enemy laser beam, $\left\|q-q_{\text {vitail }}\right\|^{2}$ is the distance of current robot pose to the desired position $\left(q_{s p p g}\right)$. When the traditional functions repulsive force reach the desired position and orientation, $\left\|q-q_{\text {vitail }}\right\|^{2}$ becomes 0 . This time, according to formula (4) shows that repulsive force is 0 , so as to ensure at the time of reaching the desired position and orientation the total potential field force is 0 . Therefore, for new potential field, all resultant force will drive robot away the enemy vital laser beam and gradually reach the desired position and orientation.

According to the various forces defined on the image space the force of the image space is:

$$
\begin{gathered}
t(q)=\beta t_{a}(q)+\left(\partial t_{i}(q)+x t_{i}(t)\right) \\
\eta+t_{j}(q) \cdot \gamma \sum_{i=1}^{k}\left(t_{i}-t_{i}^{*}\right)
\end{gathered}
$$

Where $t_{a}(q)$ is attractive, $t_{i}(q), t_{j}(q)$ and $t_{i}(q)$ respectively represent the three kinds of repulsion, and $\alpha, \beta, \chi, \lambda$ and $\mu$ are the scaling factor.

The use of $t(f)$ can get a workable trajectory in the image space. Here is the way to track the trajectory on the use of image-based visual servo control. In the $k$-th control period, the error of image feature is defined as:

$t=q_{k}^{*}-q_{k}$

Where, $q_{k}^{*}$ represents the current characteristics desired target position which selected in the generated image shot. $q_{k}$ is the position of the target feature which extracted from the visual feedback system of the $k$-th frame image.

Then, the joint space could be selected by the controller. $q=-k_{q} l_{q}+l_{r}+e-t_{d} k_{q}+k_{r}+e$

Where, $q$ is the joint space velocity sending to the robot controller, $k_{q}$ and $l_{q}$ are the control gain, and $l_{r} \quad l_{q}$ are respectively the pseudo inverse of robot and image Lie algebra matrix. The visual system block diagram is shown in Figure 1.

At this moment, because of the image-based visual servo control, it uses the Formula (7) as the controller; the system has good robustness to model error and noise disturbance. 


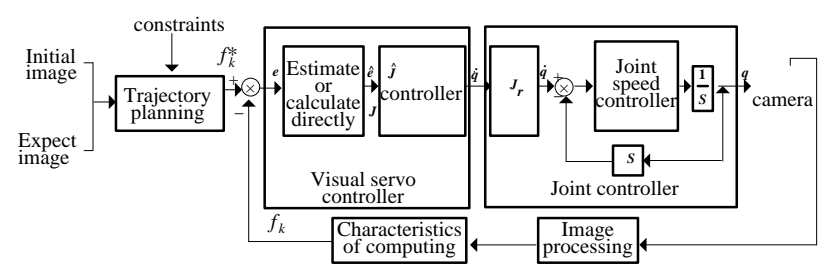

Figure1. The visual control system block diagram

In each sampling period of image, the error of image features could be calculated according to Formula (6), then the control task is to make the error e to zero, the controlling amount of joint space could be calculated by Formula (7).

\section{Experimental Results}

The contents of experimental simulation are that, to control its own robot through visual servo, and then use a laser beam to attack the laser receiver of enemy robot. There is a rectangular logo stickers in enemy disc ends which provides four feature points for visual servo, meanwhile, the enemy robots are hitting target with artificial operation. As shown in Figure 2 to Figure 4, the image error becomes zero after 120time periods, indicating that the hitting job can be accurately accomplished. As can be seen in Figure 2, the feature point has been in the camera's field of vision, it doesn't arise from the perspective phenomenon just because of the larger initial error (as shown in Figure 4).

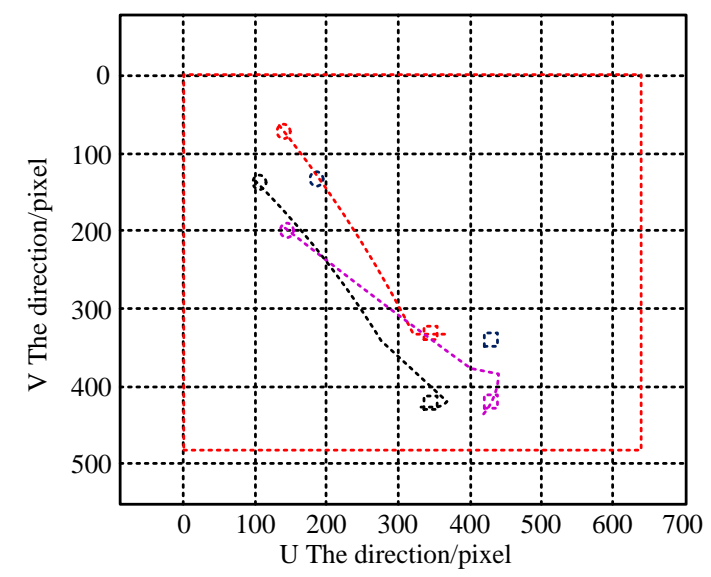

Figure2. Trajectory of feature points in image space

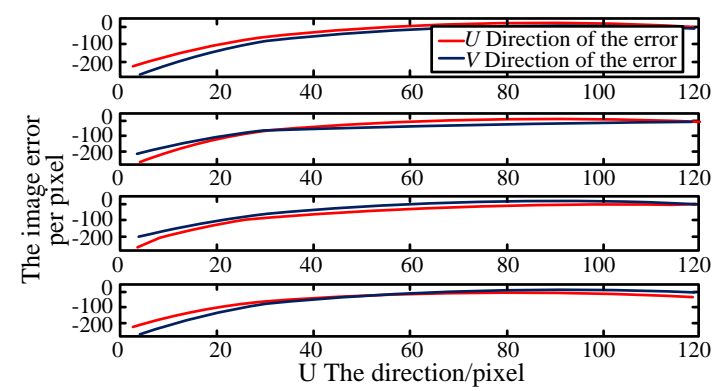

Figure3. Error of feature points in image space 


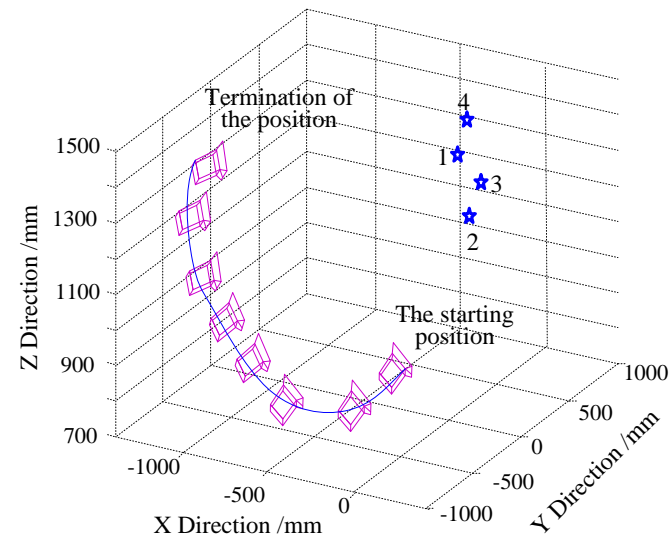

Figure4 Trajectory of camera (ends of manipulator) in Cartesian space

\section{Conclusion}

In order to further verify the correctness and effectiveness of the attack and defense method which based on trajectory planning and image visual servo control, the method is experimental analyzed in detail in the competitive network robot system. Experiments show that: the method is so simple and effective that can effectively engage targets while avoiding the other's attack.

\section{Acknowledgement}

In this paper, the research was sponsored by the Nature Science Foundation of Henan Province (Project No. 201112400450401) and Youth Fund Project of Luoyang Institute of Science and Technology (Project No. 2010QZ16).

\section{References}

[1]Huan Zhao, Kai Zhao, He Liu, Fei Yu, Improved MFCC Feature Extraction Combining Symmetric ICA Algorithm for Robust Speech Recognition, Journal of multimedia, Vol. 7, No. 1, 2012. pp: $74-81$

[2]ISO/FDIS 25178-2, Geometrical product specifications (GPS) - Surface texture: Areal - Part 2:Terms, definitions and surface texture parameters, ISO, Geneva, 2010.

[3]LI La yuan, LI Chun lin, "A multicast routing protocol with multiple QoS constraints," Journal of Software, vol. 15, No. 2, 2004, pp. 286-291.

[4]J. He, Y. Geng and K. Pahlavan, Modeling Indoor TOA Ranging Error for Body Mounted Sensors, 2012 IEEE 23nd International Symposium on Personal Indoor and Mobile Radio Communications (PIMRC), Sydney, Australia Sep. 2012 (page 682-686)

[5]S. Li, Y. Geng, J. He, K. Pahlavan,Analysis of Three-dimensional Maximum Likelihood Algorithm for Capsule Endoscopy Localization, 2012 5th International Conference on Biomedical Engineering and Informatics (BMEI), Chongqing, China Oct. 2012 (page 721-725) 